\title{
A HALL-TYPE CLOSURE PROPERTY FOR CERTAIN FITTING CLASSES
}

\author{
OWEN J. BRISON
}

(Received 27 July 1991; revised 4 February 1993)

Communicated by H. Lausch

\begin{abstract}
A closure operation connected with Hall subgroups is introduced for classes of finite soluble groups, and it is shown that this operation can be used to give a criterion for membership of certain special Fitting classes, including the so-called 'central-socle' classes.
\end{abstract}

1991 Mathematics subject classification (Amer. Math. Soc.): $20 \mathrm{D} 10$.

In this note a closure operation connected with Hall subgroups is introduced for classes of finite soluble groups. It is shown that this operation can be used to give a criterion for membership of certain special Fitting classes, namely the so-called 'central socle' classes $\mathscr{Z}_{\pi}$, and the classes $e_{\pi}\left(\mathscr{N}^{k}\right)$ : see Section 1 for definitions. Thus, for example, let $G$ be a finite soluble group and let $\sigma$ denote the set of primes which divide $|\operatorname{soc}(G)|$; we show (Theorem 2.6) that $G \in \mathscr{Z}_{\pi}$ if and only if the Hall $\tau$-subgroups of $G$ belong to $\mathscr{Z}_{\pi}$ for all sets $\tau$ of the form $\tau=\sigma \cup\{t\}$ where $t$ is a prime.

The paper has three sections. The first consists of preliminaries. In the second, the classes $\mathscr{Z}_{\pi}$ are investigated, while the classes $e_{\pi}\left(\mathscr{N}^{k}\right)$ form the subject of the third.

\section{Preliminaries}

All groups considered here will belong to the class $\mathscr{S}$ of finite soluble groups: our classes of groups are isomorphism-closed and contain all groups of order 1 . A Fitting class is a class of groups closed under the taking of subnormal subgroups and normal products; a background to Fitting class theory can be found in $[6,10]$.

If $G$ is a group and $\mathscr{F}$ is a Fitting class then $G_{\mathscr{F}}$ denotes the $\mathscr{F}$-radical of $G$,

(C) 1995 Australian Mathematical Society 0263-6115/95\$A2.00+0.00 
while $Z(G)$ denotes the centre of $G$. The set of all primes is denoted by $\mathbf{P}, p$ will always denote a prime and $\pi$ will always denote some subset of $\mathbf{P}$. Then $\pi-\operatorname{soc}(G)$ denotes the product of the minimal normal $\pi$-subgroups of $G$, while $\operatorname{soc}(G)$ denotes P-soc $(G)$. Let $\mathscr{F}$ be a Fitting class, and define classes of groups as follows:

$$
\begin{gathered}
\mathscr{Z}_{\pi}=(G \in \mathscr{S}: \pi-\operatorname{soc}(G) \leq Z(G)), \\
e_{\pi}(\mathscr{F})=\left(G \in \mathscr{S}: \text { the } G \text {-chief } \pi \text {-factors below } G_{\mathscr{F}} \text { are central in } G\right), \\
\mathscr{N}=(G \in \mathscr{S}: G \text { is nilpotent }), \\
\mathscr{S}_{\pi}=(G \in \mathscr{S}: G \text { is a } \pi \text {-group }) .
\end{gathered}
$$

In addition, we write $\mathscr{Z}=\mathscr{Z}_{\mathrm{p}}$, while (1) denotes the class of groups of order 1 .

It is well-known that both $\mathscr{Z}_{\pi}$ and $e_{\pi}(\mathscr{F})$ are Fitting classes, and that $\mathscr{Z}_{\pi}$ is subdirect-product-closed while $e_{\pi}(\mathscr{F})$ is a Fischer class: see [6] for definitions, and [12] for details. Both these families of classes, especially the former, have been extensively studied and have often been used to furnish examples or counterexamples: see, for example, $[2,4,5,7,12]$.

Write $\operatorname{Hall}_{\pi}(G)$ for the set of Hall $\pi$-subgroups of $G$, Hall $(G)$ for the set of all Hall subgroups of $G$, and $\operatorname{Syl}_{p}(G)$ for the set of Sylow $p$-subgroups of $G$. Write $C_{m}$ for the cyclic group of order $m$.

Let $\mathscr{X} \subseteq \mathscr{S}$ be a class of groups and $\mathscr{F}$ be a Fitting class. Define $\mathrm{H}_{\mathscr{F}} \mathscr{X}=$ ( $G \in \mathscr{S}: \exists X \in \mathscr{X}$ and $H \in \operatorname{Hall}(X)$ with $H \geq X_{\mathscr{F}}$ such that $G \simeq H$ ), and write $\mathrm{H} \mathscr{X}$ for $\mathrm{H}_{(1)} \mathscr{X}$. It is not hard to check that $\mathrm{H}_{\mathscr{F}}$ is a closure operation on classes of groups in the sense that (i) $\mathscr{X} \subseteq \mathrm{H}_{\mathscr{F}} \mathscr{X}$, (ii) $\mathrm{H}_{\mathscr{F}} \mathscr{X} \subseteq \mathrm{H}_{\mathscr{F}} \mathscr{Y}$ if $\mathscr{X} \subseteq \mathscr{Y}$, and (iii) $\mathrm{H}_{\mathscr{F}} \mathscr{X}=\mathrm{H}_{\mathscr{F}} \mathrm{H}_{\mathscr{F}} \mathscr{X}$. If $\mathscr{X}=\mathrm{H}_{\mathscr{F}} \mathscr{X}$, we say that $\mathscr{X}$ is $\mathrm{H}_{\mathscr{F}}$-closed, while an $\mathrm{H}$-closed class is called Hall-closed: see [1, 2, 3, 8], and the references contained therein, for results related to Hall-closure.

\section{The central-socle classes}

The section begins with Proposition 2.1, to the effect that $\mathscr{Z}_{\pi}$ is $\mathrm{H}_{\mathscr{N}}$-closed, and this is followed by Examples 2.2 to show that $\mathscr{Z}_{\pi}$ is not Hall-closed for $\pi \neq \emptyset$. A converse to Proposition 2.1 is proved as Proposition 2.5, and together these results yield a criterion, Theorem 2.6 , for membership of $\mathscr{Z}_{\pi}$. The section ends with a result, not strictly connected with the $\mathrm{H}_{\mathscr{F}}$ operation, in a similar spirit to 2.5 .

2.1 Proposition. Let $\pi \subseteq \mathbf{P}$ and let $G \in \mathscr{Z}_{\pi}$. Suppose that $H$ is a Hall subgroup of $G$ with $H \geq \operatorname{soc}(G)$. Then $H \in \mathscr{Z}_{\pi}$. Thus $\mathscr{Z}_{\pi}$ is $\mathrm{H}_{\mathscr{N}}$-closed. 
PROOF. It is easy to check that $\mathscr{Z}_{\pi}=\bigcap_{p \in \pi} \mathscr{Z}_{p}$, and so we may without loss of generality assume that $\pi=\{p\}$ for some $p \in \mathbf{P}$.

Suppose for a contradiction that $G$ is a group of minimal order subject to

(i) $G \in \mathscr{Z}_{p}$; and

(ii) there exists a Hall subgroup of $G$ which contains $\operatorname{soc}(G)$ but does not belong to $\mathscr{Z}_{p}$.

Let $H$ be a Hall subgroup of $G$ with $H \geq \operatorname{soc}(G)$ but $H \notin \mathscr{Z}_{p}$. Write $\tau=\{t \in$ $\mathbf{P}: t|| H \mid\}$; then $H \in \operatorname{Hall}_{\tau}(G), F(G) \in \mathscr{S}_{\tau}, F(G) \leq H$, and $O_{\tau^{\prime}}(G)=1$. Since $\mathscr{S}_{p^{\prime}} \subseteq \mathscr{Z}_{p}$, then $p \in \tau$. Let $M \triangleleft \cdot G$ with $F(G) \leq M$ : this is possible because $H<G$. Then $F(M)=F(G) \leq M \cap H \in \operatorname{Hall}_{\tau}(M)$, and so $M \cap H \in \mathscr{Z}_{p}$ by minimality. In particular, $H \not M$. Thus $G=M H$ and $|G: M|=q \in \tau$. Because $H \notin \mathscr{Z}_{p}$, there exists $L \cdot \unlhd H$ with $L \in \mathscr{S}_{p}$ and $L \unlhd Z(H)$. Because $F(G) \unlhd H$, then $[F(G), L] \leq F(G) \cap L \unlhd H$. Now $C_{G}(F(G)) \leq F(G)$, because $G$ is soluble, and so $F(G) \cap L>1$ because $L>1$. Since $L \cdot \unlhd H$, it follows that $L \leq F(G)$. In particular, $L \leq M \cap H$. Now $L$ is an irreducible $H$-module. Since $(M \cap H) \unlhd H$, then by Clifford's Theorem, $[9,3.4 .1]$ or $[11, \mathrm{~V} .17 .3]$, we have

$$
\left.L\right|_{(M \cap H)}=U_{1} \oplus \cdots \oplus U_{n},
$$

for some $n \in \mathbf{N}$, where each $U_{i}$ is an irreducible $(M \cap H)$-module. But this means that, as a normal subgroup of $M \cap H, L$ is a direct product of minimal normal subgroups. Thus $L \leq p-\operatorname{soc}(M \cap H)$. But $M \cap H \in \mathscr{Z}_{p}$ and so

$$
L \leq Z(M \cap H) .
$$

But $L \cdot \unlhd H$ and $L \not \Sigma Z(H)$; thus

$$
H /(M \cap H) \simeq C_{q} \text { acts faithfully and irreducibly on } L \in \mathscr{S}_{p} .
$$

In particular, $p \neq q$.

Let $J=\left\langle L^{g}: g \in G\right\rangle$, the normal closure of $L$ in $G$. We have $J \leq F(G) \leq M \cap H$ because $L \leq F(G)$. Then (1) implies that $L \leq Z(J)$. But $Z(J) \unlhd G$ and so $J=Z(J)$ is abelian and must now be a $p$-group, as it is generated by commuting conjugates of $L$.

Let $S_{1} \in \operatorname{Hall}_{\tau^{\prime}}(G)$. By orders we have $G=H S_{1}$ and $M \geq S_{1}$, whence, remembering that $L \unlhd H$, we have

$$
J=\left\langle L^{h s}: h \in H, s \in S_{1}\right\rangle=\left\langle L^{s}: s \in S_{1}\right\rangle .
$$

By the Frattini argument, using the conjugacy of Hall subgroups, we have $G=$ $M N_{G}\left(S_{1}\right)$. But $|G: M|=q$, and so there exists a $q$-element $n_{1} \in N_{G}\left(S_{1}\right)$ such that 
$G=M\left\langle n_{1}\right\rangle$. Again by Hall's Theorem, there exists $a \in G$ with $n_{1}^{a} \in H$. Write $n=n_{1}^{a} \in H \backslash M$ and $S=S_{1}^{a}$. Then $n \in N_{H}(S), G=H S$ and $J=\left\langle L^{s}: s \in S\right\rangle$. It follows that

$L$ is contained in no proper $S$-invariant subgroup of $J$.

We have $S\langle n\rangle \leq G$ because $n \in N_{H}(S)$; also, $S\langle n\rangle \in \mathscr{S}_{p^{\prime}}$ because $p \in \tau, S \in \mathscr{S}_{p}$ and $|n|=q^{\alpha}$ with $q \neq p$. Now $J$ is a normal, abelian $p$-subgroup of $G$ and so by $[9,5.2 .3]$ we have

$$
J=[J, S\langle n\rangle] \times C_{J}(S\langle n\rangle) .
$$

Since $J \unlhd G$, there exists $J^{0} \cdot \triangleleft G$ with $J^{0} \leq J$. Then $J^{0} \leq p$-soc $(G) \leq Z(G)$ and so $C_{J}(S\langle n\rangle) \geq J^{0}>1$. Thus $[J, S\langle n\rangle]<J$ by (4). But $[J, S\langle n\rangle]$ is $S\langle n\rangle$-invariant and so $S\langle n\rangle$ centralises the non-trivial group $J /[J, S\langle n\rangle]$. But then any subgroup lying between $[J, S\langle n\rangle]$ and $J$ must be $S$-invariant. By statement (3) above, it follows that $[J, S\langle n\rangle] L=J$. But then

$$
1 \neq J /[J, S\langle n\rangle]=[J, S\langle n\rangle] L /[J, S\langle n\rangle] \simeq L /(L \cap[J, S\langle n\rangle]),
$$

and since all relevant subgroups here are $\langle n\rangle$-invariant then the isomorphism is an $\langle n\rangle$-isomorphism. But $\langle n\rangle$ centralises $J /[J, S\langle n\rangle]$, and so $\langle n\rangle$ must centralise a nontrivial factor group of $L$. However, $n \in H \backslash M$ whence $H=(M \cap H)\langle n\rangle$ and so by statement (2), $L$ must be a faithful, irreducible module for $\langle n\rangle /\left\langle n^{q}\right\rangle \simeq C_{q}$, contrary to what we have just seen. This completes the proof.

2.2 EXAMPLES. The main aim of these examples is to show that $\mathscr{Z}_{\pi}$ is not Hallclosed, so that some such condition as ' $H \geq \operatorname{soc}(G)$ ' is needed in 2.1. Examples of classes (i) not $\mathrm{H}_{\mathscr{N}}$-closed, and (ii) not $\mathrm{H}_{\mathscr{S}_{\pi}}$-closed, will be given in 3.2.

(i) Suppose that $p, q$ and $r$ are distinct primes. It is well-known that there exists a group $G$ with a unique chief series whose factors have orders (reading 'from the top') of the form $p, q^{\alpha}$ and $r^{\beta}$, respectively. Then $|\operatorname{soc}(G)|=r^{\beta}$.

(a) Now suppose that $\pi$ with $\emptyset \subset \pi \subset \mathbf{P}$ (proper inclusions) is given. We show that $\mathscr{Z}_{\pi}$ is not Hall-closed. Choose $q \in \pi$ and $r \in \mathbf{P} \backslash \pi$. Then $G \in \mathscr{Z}_{\pi}$. Let $H \in \operatorname{Hall}_{(p, q)}(G)$; then $H$ has a non-central $\pi$-socle of order $q^{\alpha}$, so $H \notin \mathscr{Z}_{\pi}$.

(b) In Proposition 2.1, it is natural to ask whether the conclusion still holds if the condition ' $H \geq \operatorname{soc}(G)$ ' is replaced by ' $H \geq \pi-\operatorname{soc}(G)$ '. It need not. For take $\pi=\{p, q\}$. Then $G \in \mathscr{Z}_{\pi}$, while if $H \in \operatorname{Hall}_{\pi}(G)$ then $H \geq \pi-\operatorname{soc}(G)$ although $H \notin \mathscr{Z}_{\pi}$.

(ii) We now show that $\mathscr{Z}=\mathscr{Z}_{\mathrm{P}}$ is not Hall-closed: the above example is of no avail for this purpose. 
Let $S$ denote the group $S L(2,3)$ and let $Z$ denote $Z(S)$, the centre of $S$. Then $Z=\operatorname{soc}(S)$ has order 2 . Let $T$ denote a cyclic group of order 5 , and form the regular wreath product $W=S$ wrT (see $[11, \S I .15]$ ). We may write $W$ as a semidirect product $W=\left[S^{*}\right] T$, where $S^{*}$, the 'base group', is a direct product of 5 copies of $S$. Then $Z^{*}=Z\left(S^{*}\right)$ is the corresponding direct product of the respective centres of the 5 copies of $S$, and has order $2^{5}$. Now $\left[Z^{*}, T\right]$ has order $2^{4}$ and is normal in $W$. Write $\bar{W}=W /\left[Z^{*}, T\right]$. Then $\bar{W}$ has a unique minimal normal subgroup, namely $\bar{Z}^{*}=Z^{*} /\left[Z^{*}, T\right]$, and $\bar{Z}^{*}=Z(\bar{W})$. In particular, $\bar{W} \in \mathscr{Z}=\mathscr{Z}_{\mathrm{p}}$. But $\bar{W}$ has a Hall \{3,5\}-subgroup $H$ of order $3^{5} 5$ and $H \simeq C_{3} w r C_{5}$. Now, $C_{3}$ wr $C_{5}$ has two minimal normal subgroups: a central subgroup of order 3 and a non-central subgroup of order $3^{4}$. Thus $H \notin \mathscr{Z}$ and so $\mathscr{Z}$ is not Hall-closed.

We next prove some results converse in sense to 2.1 .

2.3 LEMMA. Suppose that $G \in \mathscr{S}$ and that $M \triangleleft \cdot G$ with $|G: M|=q$ and $M \in \mathscr{Z}_{p}$ where $p, q \in \mathbf{P}$. Suppose that $p-\operatorname{soc}(G) \leq M$. Let $H \in \operatorname{Hall}_{\tau}(G)$ where $\{p, q\} \subseteq \tau \subseteq \mathbf{P}$. Then if $H \in \mathscr{Z}_{p}$ it follows that $G \in \mathscr{Z}_{p}$.

Proof. We may suppose that $p-\operatorname{soc}(G) \neq 1$. Let $N . \unlhd G$ with $N \in \mathscr{S}_{p}$. Then $N$ is an irreducible $G$-module; thus by Clifford's Theorem, $N$ is a completely reducible $M$-module. But then $N \leq p$ - $\operatorname{soc}(M) \leq Z(M)$. Thus $M \leq C_{G}(N)$ and so $N$ is an irreducible $G / M$-module. Since $|G: M|=q \in \tau$, then $G=M H$, and so $N$ is an irreducible $H /(M \cap H)$-module. But then $N \leq p$-soc $(H) \leq Z(H)$. Thus $C_{G}(N) \geq M H=G$ and the assertion follows.

2.4 Notation. If $G \in \mathscr{S}$, write $\sigma_{G}=\{s \in \mathbf{P}: s|| \operatorname{soc}(G) \mid\}$.

2.5 Proposition. Let $G \in \mathscr{S}$ and $\pi \in \mathbf{P}$. Suppose that $\operatorname{Hall}_{\tau}(G) \subseteq \mathscr{Z}_{\pi}$ for all $\tau \subseteq \mathbf{P}$ of the form $\tau=\sigma_{G} \cup\{t\}$ where $t \in \mathbf{P}$. Then $G \in \mathscr{Z}_{\pi}$.

PROOF. It will suffice to prove that $G \in \mathscr{Z}_{p}$ for all $p \in \pi \cap \sigma_{G}$. If $\operatorname{soc}(G)=G$ there is nothing to prove and so we assume that $\operatorname{soc}(G)<G$. Let $M \triangleleft \cdot G$ with $M \geq \operatorname{soc}(G)$ and write $|G: M|=q \in \mathbf{P}$.

We claim that $\sigma_{G}=\sigma_{M}$. For suppose that $s \in \sigma_{M}$; then there exists $K \cdot \unlhd M$ with $K \in \mathscr{S}_{s}$. The normal closure $K^{G}$ satisfies $\mathscr{S}_{s} \ni K^{G} \leq M$, and so there exists $L \cdot \unlhd G$ with $L \leq K^{G}$. Thus $s \in \sigma_{G}$. Next suppose that $s \in \sigma_{G}$. Then there exists $K \cdot \unlhd G$ with $K \in \mathscr{S}_{s}$, and $K \leq M$ because $M \geq \operatorname{soc}(G)$. Thus there exists $L \cdot \unlhd M$ with $L \leq K$, whence $s \in \sigma_{M}$, and $\sigma_{G}=\sigma_{M}$.

Let $\tau$ be of the form $\tau=\sigma_{M} \cup\{t\}=\sigma_{G} \cup\{t\}$, where $t \in \mathbf{P}$. Let $H_{1} \in \operatorname{Hall}_{\tau}(M)$ and let $H \in \operatorname{Hall}_{\tau}(G)$ with $H_{1}=H \cap M$. By hypothesis, $H \in \mathscr{Z}_{\pi}$ and so $H_{1} \in \mathscr{Z}_{\pi}$. By the minimality of $G$, it follows that $M \in \mathscr{Z}_{\pi}$. 
Now write $\tau_{0}=\sigma_{G} \cup\{q\}$ and fix $H \in \operatorname{Hall}_{\tau_{0}}(G)$. Let $p \in \pi \cap \sigma_{G}$ be arbitrary. Then $H \in \mathscr{Z}_{p}, M \in \mathscr{Z}_{p}$, and $\{p, q\} \subseteq \tau_{0}$; it follows from Lemma 2.3 that $G \in \mathscr{Z}_{p}$, and the proof is complete.

Putting together Propositions 2.1 and 2.5, we obtain the promised criterion for membership of the central-socle classes as follows.

2.6 THEOREM. Let $G \in \mathscr{S}$ and $\pi \subseteq \mathbf{P}$. Then $G \in \mathscr{Z}_{\pi}$ if and only if $\operatorname{Hall}_{\tau}(G) \subseteq \mathscr{Z}_{\pi}$ for all $\tau \subseteq \mathbf{P}$ of the form $\tau=\sigma_{G} \cup\{t\}$ with $t \in \mathbf{P}$.

We now give another result in the spirit of 2.5.

2.7 Proposition. Let $G \in \mathscr{S}$ and $\pi \subseteq \mathbf{P}$. Suppose that $\operatorname{Hall}_{\tau}(G) \subseteq \mathscr{Z}_{\pi}$ for all sets of primes $\tau$ with $|\tau| \leq 2$. Then $G \in \mathscr{Z}_{\pi}$.

PROOF. Because $\mathscr{Z}_{\pi}=\bigcap_{p \in \pi} \mathscr{Z}_{p}$, we may without loss of generality assume that $\pi=\{p\}$. Suppose for a contradiction that $G$ is a counterexample of minimal order. Then $p-\operatorname{soc}(G)<G$ and there exists $M \unlhd \cdot G$ with $M \geq p$-soc $(G)$. If $\tau \subseteq \mathbf{P}$ with $|\tau|=2$ and if $H \in \operatorname{Hall}_{\tau}(M)$, then $H=M \cap H_{1}$ where $H_{1} \in \operatorname{Hall}_{\tau}(G)$, and so $H \in \mathscr{Z}_{p}$. Thus $M \in \mathscr{Z}_{p}$ by minimality. Write $|G: M|=q \in \mathbf{P}$. Now the Hall $\{p, q\}$-subgroups of $G$ belong to $\mathscr{Z}_{p}$ by hypothesis, and the result follows from Lemma 2.3.

\section{The classes $e_{\pi}\left(\mathscr{N}^{k}\right)$}

This section has a similar structure to Section 2. It is proved in Proposition 3.1 that $e_{\pi}\left(\mathscr{N}^{k}\right)$ is $\mathrm{H}_{\mathscr{N}}$-closed, and this is followed by some relevant examples (3.2). Proposition 3.3 is a converse to Proposition 3.1, and together these results yield a criterion, Theorem 3.4, for membership of the classes $e_{\pi}\left(\mathscr{N}^{k}\right)$. Again the section finishes with a result, Proposition 3.5, not strictly connected with the $\mathrm{H}_{\mathscr{F}}$ operation, being an analogue for certain classes $e_{\pi}(\mathscr{F})$ of Proposition 2.7.

3.1 Proposition. Let $\pi \subseteq \mathbf{P}$ and $k \in \mathbf{N}, k \geq 0$. Let $G \in e_{\pi}\left(\mathscr{N}^{k}\right)$. Suppose that $H$ is a Hall subgroup of $G$ with $H \geq G_{\mathscr{F}^{k}}$. Then $H \in e_{\pi}\left(\mathscr{N}^{k}\right)$. It follows that $\boldsymbol{e}_{\pi}\left(\mathscr{N}^{k}\right)$ is $\mathrm{H}_{\mathscr{N}^{k}}$-closed.

PROOF. Because $e_{\pi}\left(\mathscr{N}^{k}\right)=\bigcap_{p \in \pi} e_{p}\left(\mathscr{N}^{k}\right)$, we may without loss of generality assume that $\pi=\{p\}$ where $p \in \mathbf{P}$.

The proof is by induction on $k$. If $k=0$ then $\mathscr{N}^{k}=1$ and $e_{p}(1)=\mathscr{S}$; the conclusion clearly holds in this case. We thus suppose that the result holds for all $G_{0} \in e_{p}\left(\mathscr{N}^{k_{0}}\right)$ for all $k_{0}<k$, and for all $G_{1} \in e_{p}\left(\mathscr{N}^{k}\right)$ with $\left|G_{1}\right|<|G|$. 
Write $\tau=\{q \in \mathbf{P}: q|| H \mid\}$; then $H \in \operatorname{Hall}_{\tau}(G)$. If $A$ is a group, write $A_{j}=A_{\mathcal{N}^{\prime}}$, the $\mathscr{N}^{j}$-radical of $A$; then $G_{k} \in \mathscr{S}_{\tau}$ and $G_{k} \leq O_{\tau}(G) \leq H$, where $O_{\tau}(G)$ denotes the $\mathscr{S}_{\tau}$-radical of $G$. Since $\mathscr{S}_{p^{\prime}} \in e_{p}\left(\mathscr{N}^{k}\right)$, then $H \in e_{p}\left(\mathscr{N}^{k}\right)$ if $p \notin \tau$, and so we may without loss assume that $p \in \tau$.

Choose $M \triangleleft \cdot G$ with $M \geq G_{k}$ and write $|G: K|=q \in \mathbf{P}$. Then $M \in e_{p}\left(\mathscr{N}^{k}\right)$, $M \cap H \in \operatorname{Hall}_{\tau}(M)$ and $M_{k}=G_{k} \leq M \cap H$. By the induction hypothesis we have $M \cap H \in e_{p}\left(\mathscr{N}^{k}\right)$; in particular, $M \cap H \neq H$ and so $G=M H$. Further, all $M \cap H$-chief $p$-factors below $(M \cap H)_{k}$ are $M \cap H$-central, Since $M \cap H \unlhd H$ then by Clifford's Theorem, any $H$-chief $p$-factor, $X / Y$ say, below $(M \cap H)_{k}$ is completely reducible as an $M \cap H$-module and, being then a sum of $M \cap H$-trivial modules, must itself be $M \cap H$-trivial. Thus,

The $H$-chief $p$-factors below $(M \cap H)_{k}$ are $M \cap H$-central.

There are now two cases to consider.

Case (I). Suppose that $H_{k} \not M$; then $H=(M \cap H) H_{k}$. Let $X / Y$ be an $H$-chief p-factor in $H_{k}$ in an $H$-chief series which refines $H \geq H_{k} \geq H_{k-1} \geq 1$. By the Jordan-Hölder theorem, we may restrict attention to a fixed chief series.

We firstly claim that $X / Y$ is trivial as an $M \cap H$-module. If $X \leq(M \cap H)_{k}=$ $M \cap H_{k}$, then $X / Y$ is $M \cap H$-central by (1). If $Y \not Z M$ then $X / Y \simeq_{H}(X \cap M) /(Y \cap M)$; the latter is still $H$-chief and so again is $M \cap H$-trivial by (1). In the remaining case we have $Y \leq M, X \leq M$ and $Y=X \cap M$; then we have $[X, M \cap H] \leq X \cap M=Y$ and again $X / Y$ is $M \cap H$-trivial; this justifies our claim.

Suppose that $X / Y$ lies below $H_{k-1}$; then $X / Y$ is $H$-central because $H \in e_{p}\left(\mathscr{N}^{k-1}\right)$ by the induction hypothesis and the fact that $e_{p}\left(\mathscr{N}^{k}\right) \subseteq e_{p}\left(\mathscr{N}^{k-1}\right)$. Suppose, on the other hand, that $X / Y$ lies between $H_{k}$ and $H_{k-1}$. By Clifford's Theorem, $X / Y$ is completely reducible as an $H_{k}$-module and so must be a sum of $H_{k}$-trivial submodules because $H_{k} / H_{k-1}$ is nilpotent; thus $X / Y$ is a trivial $H_{k}$-module. But $H=(M \cap H) H_{k}$, and since $X / Y$ is trivial for $M \cap H$, it must be trivial for $H$. It follows that $H \in$ $e_{p}\left(\mathscr{N}^{k}\right)$, as required.

Case (II). Suppose now that $H_{k} \leq M$; then $H_{k}=(M \cap H)_{k}$. Now $G_{k} \leq O_{\tau}(G) \cap$ $H_{k} \leq\left(O_{\tau}(G)\right)_{k} \leq G_{k}$, whence $G_{k}=O_{\tau}(G) \cap H_{k}$.

Let $P \in \operatorname{Syl}_{p}\left(H_{k}\right)$, and write $J=\left\langle P^{g}: g \in G\right\rangle$, the normal closure of $P$ in $G$; note that $J \leq M$. Let $R$ be a Hall $p$-complement in $G_{k}$; then $\bar{R}=R G_{k-1} / G_{k-1}$ is the unique $p$-complement in $G_{k} / G_{k-1} \in \mathscr{N}$, and so $\bar{R} \unlhd G / G_{k-1}$. Now $R \in H_{k}$ and so, since $H_{k} / H_{k-1} \in \mathscr{N}$, we have $[R, P] \leq H_{k-1}$. But $[R, P] \leq G_{k}$ because $R \leq G_{k} \unlhd G$, and so

$$
[R, P] \leq G_{k} \cap H_{k-1}=O_{\tau}(G) \cap H_{k} \cap H_{k-1}=G_{k-1} .
$$

But then $P \leq C_{G}(\bar{R}) \unlhd G$ and so $J \leq C_{G}(\bar{R}) \cap M$. Now let $x \in J$ be a $p^{\prime}$-element. The $G$-chief $p$-factors between $G_{k}$ and $G_{k-1}$ are $G$-central because $G \in e_{p}\left(\mathscr{N}^{k}\right)$, and 
so are centralised by $x$. But then $x$, being a $p^{\prime}$-element, must centralise the Sylow $p$ subgroup of $G_{k} / G_{k-1}$, by [9,5.3.2]. But $x \in J$ already centralises the $p$-complement $R G_{k-1} / G_{k-1}$ of $G_{k} / G_{k-1}$, and so $x$ centralises $G_{k} / G_{k-1}$. But $G_{k} / G_{k-1}$ is the Fitting subgroup of $G / G_{k-1}$, and so $x \in G_{k}$ by [9,6.1.3]. But this implies that $J G_{k} / G_{k}$ must be a $p$-group. Since $G_{k} \in \mathscr{S}_{\tau}$ and $p \in \tau$, it follows that $J \in \mathscr{S}_{\pi}$. But now $J \leq O_{\tau}(G)$ and $P \leq O_{\tau}(G) \cap H_{k}=G_{k}$. But then $P \in \operatorname{Syl}_{p}\left(G_{k}\right)$ and so $p \nmid\left|H_{k}: G_{k}\right|$.

Let $\mathscr{C}_{0}$ be a $G$-chief series between $G_{k}$ and 1 , and let $\mathscr{C}$ be an $H$-chief series which refines $H_{k} \geq G_{k} \geq 1$ and which refines $\mathscr{C}_{0}$ below $G_{k}$. Now all the $G$-chief $p$-factors in $\mathscr{C}_{0}$ are $G$-central because $G \in e_{p}\left(\mathscr{N}^{k}\right)$; thus they all have order $p$ and so must be $H$-chief; moreover, they give us all the $p$-factors in $\mathscr{C}$ because $p \nmid\left|H_{k}: G_{k}\right|$. But now $H \in e_{p}\left(\mathscr{N}^{k}\right)$, and the proof is complete.

3.2 ExAmples. (i) This example is to show that $e_{p}\left(\mathscr{N}^{2}\right)$ is not $\mathbf{H}_{\mathscr{N}}$-closed. Let $p, q, r$ and $s$ be distinct primes. There exists a group $G$ with a unique chief series whose factors have orders (reading 'from the top') of the form $q, p^{\alpha}, r^{\beta}$ and $s^{\gamma}$ respectively. Then $G \in e_{p}\left(\mathscr{N}^{2}\right)$ because $\left|G_{\mathscr{N}^{2}}\right|=s^{\gamma} r^{\beta}$. Let $H \in \operatorname{Hall}(G)$ with $|H|=s^{\gamma} p^{\alpha} q$. Then $\left|H_{\mathscr{N}^{2}}\right|=s^{\gamma} p^{\alpha}$ and $H \notin e_{p}\left(\mathscr{N}^{2}\right)$. However, $H \geq G_{\mathscr{N}}$, and so $e_{p}\left(\mathscr{N}^{2}\right)$ is not $\mathrm{H}_{\mathscr{N}}$-closed.

(ii) This example shows that $e_{p}\left(\mathscr{S}_{\pi}\right)$ is not $\mathrm{H}_{\mathscr{S}_{\pi}}$-closed when $\pi \subset \mathbf{P}$ with $|\pi| \geq 2$. Let $G$ be the group of Example 2.2(i) with $\{p, q\} \subseteq \pi, r \notin \pi$, and $H \in \operatorname{Hall}_{\pi}(G)$. Then $H \geq O_{\pi}(G)=1$. Now $G \in e_{q}\left(\mathscr{S}_{\pi}\right)$ while $H \notin e_{q}\left(\mathscr{S}_{\pi}\right)$. Thus 3.1 is not valid if we replace $\mathscr{N}^{k}$ by an arbitrary Fitting class $\mathscr{F}$.

The next result is an analogue of Proposition 2.5, being converse in sense to 3.1; it is valid for arbitrary $e_{\pi}(\mathscr{F})$ and not just for the classes $e_{\pi}\left(\mathscr{N}^{k}\right):$ as we have just seen, 3.1 is not valid for arbitrary $\boldsymbol{e}_{\pi}(\mathscr{F})$.

3.3 Proposition. Let $G \in \mathscr{S}$ and $\pi \subseteq \mathbf{P}$. Let $\mathscr{F}$ be a Fitting class. Suppose that $\operatorname{Hall}_{\tau}(G) \subseteq e_{\pi}(\mathscr{F})$ for all $\tau \subseteq \mathbf{P}$ of the form $\tau=\rho_{G} \cup\{t\}$ where $t \in \mathbf{P}$ and $\rho_{G}=\left\{s \in \mathbf{P}: s|| G_{\mathscr{F}} \mid\right\}$. Then $G \in e_{\pi}(\mathscr{F})$.

PROOF. Suppose for a contradiction that $G$ is a counterexample of minimal order. Then $G_{\mathscr{F}}<G$ as otherwise $\sigma_{G}$ contains all primes dividing $|G|$ and so $G \in e_{p}(\mathscr{F})$ by hypothesis. Let $M \triangleleft \cdot G$ with $M \geq G_{\mathscr{F}}$, and write $|G: M|=q$. Then $M_{\mathscr{F}}=G_{\mathscr{F}}$, and so $\rho_{M}=\rho_{G}$. If $H \in \operatorname{Hall}_{\tau}(M)$ then $H=H_{1} \cap M$ for some $H_{1} \in \operatorname{Hall}_{\tau}(G)$ and so $M \in e_{\pi}(\mathscr{F})$ by minimality. Because $G \notin e_{\pi}(\mathscr{F})$, there exists a $G$-chief $\pi$-factor $X / Y$ below $G_{\mathscr{F}}$ which is not $G$-central. By Clifford's Theorem, $X / Y$ is completely reducible as an $M$-module, and so $X / Y$ is $M$-central because $M \in e_{\pi}(\mathscr{F})$. Thus $X / Y$ is faithful and irreducible for $G / M \simeq C_{q}$. Let $H \in \operatorname{Hall}_{\tau}(G)$ where $\tau=\rho_{G} \cup\{q\}$. Then $G=M H$. Thus $X / Y$ is faithful and irreducible for $H /(H \cap M) \simeq G / M$, and 
so is non-trivial for $H$. Now $H \geq H_{\mathscr{F}} \geq G_{\mathscr{F}} \geq X \geq Y$, and so $X / Y$ is $H$-central because $H \in e_{\pi}(\mathscr{F})$, in contradiction to the preceding statement. The result follows.

Putting together Propositions 3.1 and 3.3, we obtain our criterion for membership of the classes $e_{\pi}\left(\mathscr{N}^{k}\right)$ as follows.

3.4 TheOREM. Let $G \in \mathscr{S}, \pi \subseteq \mathbf{P}$ and $k \in \mathbf{N}, k \geq 0$. Then $G \in e_{\pi}\left(\mathscr{N}^{k}\right)$ if and only if $\operatorname{Hall}_{\tau}(G) \subseteq e_{\pi}\left(\mathscr{N}^{k}\right)$ for all $\tau \subseteq \mathbf{P}$ of the form $\tau=\rho_{G} \cup\{t\}$ where $t \in \mathbf{P}$ and $\rho_{G}=\left\{s \in \mathbf{P}: s|| G_{\mathscr{N}^{k}} \mid\right\}$.

The next result is an analogue of Proposition 2.7 for the classes $e_{\pi}(\mathscr{F})$.

3.5 Proposition. Let $G \in \mathscr{S}$ and $\pi \subseteq$ P. Let $\mathscr{F}$ be a Hall-closed Fitting class. Suppose that $\operatorname{Hall}_{\tau}(G) \subseteq e_{\pi}(\mathscr{F})$ for all $\tau \subseteq \mathbf{P}$ with $|\tau| \leq 2$. Then $G \in e_{\pi}(\mathscr{F})$.

Proof. The proof is by induction on $|G|$, the result being trivial if $|G|=1$. If $M \triangleleft \cdot G$ and $\tau \subseteq \mathbf{P}$ with $|\tau| \leq 2$ then $\operatorname{Hall}_{\tau}(M) \subseteq e_{\pi}(\mathscr{F})$ and so $M \in e_{\pi}(\mathscr{F})$ by induction. It follows that $G$ contains a unique maximal normal subgroup, which we call $M$; then $M \geq G^{\prime}$ and $|G: M|=q \in \mathbf{P}$. Let now $X / Y$ be a $G$-chief $\pi$-factor below $G_{F}$. If $X \not \leq M$ then $X=G$ and $Y=M$ by the unicity of $M \triangleleft \cdot G$, and then $X / Y$ is certainly $G$-central. Suppose that $X \leq M$. Then $X / Y$ is below $M_{\mathscr{F}}$, and by Clifford's Theorem must be $M$-central. Now $X / Y \in \mathscr{S}_{p}$ for some $p \in \pi$. Let $H \in \operatorname{Hall}_{\tau}(G)$ where $\tau=\{p, q\}$. Then $G=M H$ and $X / Y$ is a module for $H / H \cap M \simeq G / M$. But $X \leq Y H$ and so $X=X \cap T H=Y(X \cap H)$, whence

$$
X / Y \simeq_{H}(X \cap H) /(Y \cap H)
$$

Now $M_{\mathscr{F}} \cap H \in \operatorname{Hall}_{\tau}\left(M_{\mathscr{F}}\right) \subseteq \mathscr{F}$, the final inclusion because $\mathscr{F}$ is Hall-closed, and so $X \cap H \leq M_{\mathscr{F}} \cap H \leq H_{\mathscr{F}}$. But $H \in e_{\pi}(\mathscr{F})$, and it follows that $X / Y$ is $H$-central and thus $G$-central, as required.

\section{Acknowledgements}

Proposition 2.1 and the case $k=1$ of Proposition 3.1 appear in the author's Ph.D. thesis [1]; we wish to thank Dr. T. O. Hawkes for his supervision and The Royal Commission for the Exhibition of 1851 for their financial support of that thesis. We also wish to acknowledge partial support for the balance of this article from the "Centro de Algebra da Universidade de Lisboa". 


\section{References}

[1] O. J. Brison, On the theory of Fitting classes of finite groups (Ph.D. Thesis, University of Warwick, 1978).

[2] — 'Hall-closure and products of Fitting classes', J. Austral. Math. Soc. (Series A) 32 (1982), 145-164.

[3] _ _ 'A criterion for the Hall-closure of Fitting classes', Bull. Austral. Math. Soc. 23 (1981), $351-365$.

[4] R. A. Bryce and J. Cossey, 'Metanilpotent Fitting classes', J. Austral. Math. Soc. 17 (1974), 285-304.

[5] - 'Subdirect product closed Fitting classes', in: Proceedings of the second international conference on the theory of groups, Canberra (1973), Lecture Notes in Math. 372 (Springer, Berlin, 1974) pp. 158-164,

[6] J. Cossey, 'Classes of finite soluble groups', in: Proceedings of the second international conference on the theory of groups, Canberra (1973), Lecture Notes in Math. 372 (Springer, Berlin, 1974) pp. 226-237,

[7] — 'Products of Fitting classes', Math. Z. 141 (1975), 289-295.

[8] E. Cusack, 'Normal Fitting classes and Hall subgroups', Bull. Austral. Math. Soc, 21 (1980), 229-236.

[9] D. Gorenstein, Finite groups, 2nd edition, (Chelsea, New York, 1980).

[10] T. O. Hawkes, 'Finite soluble groups', in: Group theory: Essays for Philip Hall (Academic Press, London, 1984) pp. 13-60.

[11] B. Huppert, Endliche Gruppen I (Springer, Berlin, 1967).

[12] F. P. Lockett, On the theory of Fitting classes of finite soluble groups (Ph.D. Thesis, University of Warwick, 1971).

Departamento de Matemática

Faculdade de Ciências

Rua Ernesto de Vasconcelos, Bloco C1, Piso 3

1700 Lisboa, Portugal 